\title{
Hipertensión arterial y dislipemia
}

\author{
Dres. María Noel Rivero, Luca Quiroz, Paola Spósito, Álvaro Huarte
}

\section{Resumen}

Introducción: la enfermedad cardiovascular representa mundialmente la principal causa de muerte resultando imprescindible entonces un abordaje multifactorial mediante el cálculo del riesgo cardiovascular (RCV). Múltiples series a nivel mundial han demostrado una alta asociación entre hipertensión arterial (HTA) y dislipemia, en algunas superiores al 50\%. El objetivo de este estudio es determinar la asociación entre ambas variables con el fin de profundizar en la estratificación de riesgo del paciente hipertenso.

Materiales y métodos: se realizó un estudio analítico, observacional, de tipo transversal, que incluyó a todos los pacientes que asistieron a la policlínica de HTA de nuestro centro entre el 2 de marzo de 2015 y el 2 de octubre de 2019 inclusive.

Resultados: se incluyeron 103 pacientes. El 75,7\% (n=78) presentó dislipemia. Se destaca que el 68,9\% (n=71) presenta HTA nocturna. Se estableció una asociación estadísticamente significativa entre dislipemia e HTA nocturna, con $\mathrm{OR}=3,364$. En cuanto al perfil lipídico, predomina la dislipemia mixta en un 37,9\% ( $\mathrm{n}=39$ ).

Conclusiones: existe una alta relación entre HTA y dislipemia. La HTA nocturna y el patrón non dipper asocian mayor RCV.

Palabras clave: HIPERTENSIÓN ARTERIAL

HIPERTENSIÓN ARTERIAL NOCTURNA

PATRÓN DE VARIABILIDAD

DISLIPEMIA

\section{Arterial hypertension and dyslipidemia}

\section{Summary}

Introduction: cardiovascular disease represents the main cause of death worldwide. A multifactorial approach is essential, by calculating cardiovascular risk. Series worldwide have shown a high association between hypertension and dyslipidemia, in some of them higher than $50 \%$. Our objective is to determine the association between both variables in order to improve the risk stratification of the hypertensive patient.

Materials and methods: an analytical, observational, cross-sectional study was carried out, which included all the patients who attended the hypertension polyclinic between March 2, 2015 and October 2, 2019 inclusive.

Results: $75.7 \%(\mathrm{n}=78)$ of the patients presented dyslipidemia. It is noteworthy that $68.9 \%(\mathrm{n}=71)$ have nocturnal hypertension. A statistically significant association between dyslipidemia and nocturnal hypertension was established, with an $\mathrm{OR}=3.364$. Regarding the lipid profile, mixed dyslipidemia predominates in $37.9 \%$.

Conclusions: there is a high relationship between hypertension and dyslipidemia. Nocturnal hypertension and the non-dipper pattern are associated with cardiovascular risk.

Key words: $\quad$ ARTERIAL HYPERTENSION

NOCTURNAL HYPERTENSION

VARIABILITY PATTERN

DYSLIPIDEMIA

Hospital Maciel. Montevideo, Uruguay.

Correspondencia: Dra. María Noel Rivero. Correo electrónico: marianoelriv.28@gmail.com.

Los autores declaran no presentar conflictos de intereses.

El presente estudio no tiene fuentes de financiamiento.

Recibido Ago 27, 2020; aceptado Oct 22, 2020 


\section{Hipertensão arterial e dislipidemia}

\section{Resumo}

Introdução: as doenças cardiovasculares representam a principal causa de morte no mundo. Uma abordagem multifatorial é essencial, calculando o risco cardiovascular. As séries em todo o mundo demonstraram uma alta associação entre hipertensão e dislipidemia, em alguns superiores a 50\%. Nosso objetivo é determinar a associação entre as duas variáveis, a fim de melhorar a estratificação de risco do paciente hipertenso.

Materiais e métodos: foi realizado um estudo analítico, observacional e transversal, que incluiu todos os pacientes que compareceram à policlínica hipertensão entre 2 de março de 2015 e 2 de outubro de 2019 inclusive.

Resultados: 75,7\% $(\mathrm{n}=78)$ dos pacientes apresentaram dislipidemia. Vale ressaltar que 68,9\% (n=71) possuem hipertensão noturna. Foi estabelecida associação estatisticamente significante entre dislipidemia e hipertensão noturna, com $\mathrm{OR}=3,364$. Quanto ao perfil lipídico, a dislipidemia mista predomina em 37,9\%.

Conclusões: existe uma alta relação entre hipertensão e dislipidemia. A hipertensão noturna e o padrão non dipper estão associados ao risco cardiovascular.

Palavras chave:

HIPERTENSÃO ARTERIAL
HIPERTENSÃO NOTURNA
PADRÃO DE VARIABILIDADE
DISLIPIDEMIA

¿Qué aporta este estudio al conocimiento actual?

Existe una fuerte asociación entre hipertensión arterial y dislipemia. La interrelación de los factores de riesgo cardiovascular explica la elevada morbimortalidad de los pacientes con ambas afecciones. Diversos estudios internacionales demostraron la asociación entre estas variables, siendo el objetivo del estudio actual determinar las características de la población que se asiste en la Policlínica de Hipertensión arterial del Hospital Maciel y evaluar la asociación entre hipertensión y dislipemia en nuestro medio.

\section{Introducción}

La hipertensión arterial (HTA) es la principal causa evitable de enfermedad cardiovascualr (ECV) y de mortalidad por cualquier causa a nivel mundial ${ }^{(1,2)}$.

No obstante, raramente se presenta sola, sino que coexiste con otros factores de riesgo cardiovascular (FRCV) como son: diabetes mellitus, dislipemia, obesidad y tabaquismo. La compleja interacción metabólica y fisiopatológica que comparten los citados FRCV influye en el pronóstico final del paciente $^{(3)}$. Por esta razón, para la toma de decisiones terapéuticas, es imprescindible la estratificación de $\mathrm{RCV}^{(2)}$.

Dada la compleja interacción que existe entre los distintos FRCV, la decisión terapéutica debe realizarse en base a un abordaje integral con modificación conjunta de los mismos. Para ello es imprescindible en el contacto inicial estratificar el RCV. Las guías europeas de HTA y dislipemia utilizan la escala de riesgo Systemic Coronary Risk Estimation (SCORE), integrada a otras variables modificado- ras del riesgo como son: lesión de órgano diana y presencia de ECV. Las situaciones que clasifican a los pacientes en alto o muy alto riesgo son: HTA grado III, hipercolesterolemia con colesterol unido a lipoproteínas de baja intensidad (LDLc) $>190 \mathrm{mg} / \mathrm{dl}$, diabetes mellitus (DM), enfermedad renal crónica (ERC) estadio III o más, y ECV establecida y documentada $^{(4)}$.

Con respecto a los FRCV, numerosas series han demostrado una alta asociación entre HTA y dislipemia, llegando en algunas de ellas al $50 \%(5)$.

El objetivo del presente estudio es evaluar la asociación entre HTA y dislipemia en la población de pacientes que se asisten en la policlínica de HTA del Hospital Maciel.

\section{Método}

Se realizó un estudio analítico, observacional, de tipo transversal, que incluyó a todos los pacientes que asistieron a la policlínica de HTA en el período comprendido entre el 2 de marzo de 2015 y el 2 de octubre de 2019 inclusive. La policlínica de HTA del Hospital Maciel asiste pacientes derivados por médicos generales, internistas o cardiólogos con mal control de cifras de presión arterial (PA) o por complicaciones vinculadas a la misma. La población incluida inicialmente fue de 207 pacientes. Se excluyeron aquellos que no contaban con perfil lipídico y monitoreo ambulatorio de la PA (MAPA) al momento de la recolección de datos. La población final fue de 103 pacientes.

Los datos se recogieron de la historia clínica electrónica diseñada por el equipo de HTA, donde se ingresan los antecedentes personales, comorbilidades y estudios realizados para la estratificación del paciente 
Tabla 1. Características generales de la población estudiada.

\begin{tabular}{|c|c|c|}
\hline & $\begin{array}{c}\text { Frecuencia } \\
\text { absoluta }\end{array}$ & $\begin{array}{l}\text { Frecuencia } \\
\text { relativa } \%\end{array}$ \\
\hline \multicolumn{3}{|l|}{ Sexo } \\
\hline Femenino & 71 & 68,9 \\
\hline Masculino & 32 & 31,1 \\
\hline \multicolumn{3}{|l|}{ Dislipemia } \\
\hline Ausencia & 25 & 24,3 \\
\hline Hipercolesterolemia & 20 & 19,4 \\
\hline Hipertrigliceridemia & 19 & 18,4 \\
\hline Mixta & 39 & 37,9 \\
\hline \multicolumn{3}{|l|}{ MAPA } \\
\hline Dipper & 51 & 49,5 \\
\hline Non dipper & 40 & 38,8 \\
\hline Riser & 8 & 7,8 \\
\hline Extreme dipper & 4 & 3,9 \\
\hline HTA nocturna & 71 & 68,9 \\
\hline Diabetes mellitus & 24 & 23,3 \\
\hline \multicolumn{3}{|l|}{ IMC } \\
\hline Normopeso & 12 & 11,7 \\
\hline Sobrepeso & 33 & 32,0 \\
\hline Obeso & 58 & 56,3 \\
\hline Tabaquismo & 35 & 34,0 \\
\hline
\end{tabular}

hipertenso incluyendo el monitoreo ambulatorio de la presión arterial (MAPA), así como para valoración de daño de órgano blanco. Los resultados de paraclínica se buscaron en el sistema informático "Intralab" utilizado por el laboratorio del Hospital Maciel.

Se presentan frecuencias absolutas y relativas para la descripción de variables cualitativas y medidas de resumen para las variables continuas. El estudio de asociación se realizó con test Chi cuadrado o exacto de Fisher en los casos necesarios. De encontrarse asociación, se calculó la correspondiente medida de riesgo (Odds ratio) con su Intervalo de Confianza al 95\%. El nivel de significación se fijó en 0,05 . Como software estadístico en el presente análisis se utilizó STATA v. 12.0.

\section{Definición de variables}

HTA: PA sistólica (PAS) $\geq 140 \mathrm{mmHg}$ y/ o PA diastólica $(\mathrm{PAD}) \geq 90 \mathrm{mmHg}$, registradas en tres mediciones separadas 1-2 minutos $^{(2)}$.
MAPA: es el monitoreo ambulatorio de la PA mediante un tensiómetro portátil que recoge información durante 24 horas. Su lectura evidencia la variabilidad de la PA diurna y nocturna durante las diferentes actividades que realiza el paciente, clasificando la HTA en diferentes patrones de variabili$\operatorname{dad}^{(2)}$.

Patrón dipper: patrón normal y fisiológico. Se define como un descenso de la PA media nocturna entre 10\%-20\% con respecto a la PA media diurna ${ }^{(2)}$.

Patrón non dipper: descenso atenuado de la PA media nocturna entre $0 \%-10 \%$ con respecto a la PA media diurna ${ }^{(2)}$.

Patrón dipper extremo: descenso de la PA media nocturna $>20 \%$ con respecto a la PA media diurna ${ }^{(2)}$.

Patrón riser: aumento de la PA media nocturna a valores superiores a la media diurna ${ }^{(2)}$.

HTA nocturna: $\mathrm{PA} \geq 120$ y/o $70 \mathrm{mmHg}$ durante el sueño o descanso nocturno ${ }^{(2)}$.

Tabaquismo: haber fumado por lo menos un cigarrillo en los últimos 6 meses $^{(6)}$.

DM: dos determinaciones de glicemia en ayunas $>126 \mathrm{mg} /$ dl o prueba de tolerancia oral a glucosa $>200 \mathrm{mg} / \mathrm{dl}$ a las $2 \mathrm{~h}$; o una determinación de más de $200 \mathrm{mg} / \mathrm{dl}$ en presencia de síntomas ${ }^{(7)}$.

Normopeso: índice de masa corporal (IMC) entre 18,5-24,9. Sobrepeso: IMC entre 25 y 29,9 . Obesidad: $\mathrm{IMC} \geq 30^{(8)}$.

Hipercolesterolemia: colesterol total $>190$ $\mathrm{mg} / \mathrm{dl}$ y/o LDLc $>115 \mathrm{mg} / \mathrm{dl}$ y/o colesterol unido a lipoproteínas de alta densidad (HDLc) $<40 \mathrm{mg} / \mathrm{dl}$ (hombres) y $<46 \mathrm{mg} / \mathrm{dl}$ (mujeres).

Hipertrigliceridemia: triglicéridos $>150 \mathrm{mg} / \mathrm{dl}$.

Dislipemia mixta: colesterol total $>190 \mathrm{mg} / \mathrm{dl}$ y/o LDLc $>115 \mathrm{mg} / \mathrm{dl}$ y/o HDLc $<40 \mathrm{mg} / \mathrm{dl}$ (hombres) y $<46 \mathrm{mg} / \mathrm{dl}$ (mujeres) y triglicéridos $>150$ $\mathrm{mg} / \mathrm{dl}^{(9)}$.

\section{Resultados}

Se estudiaron un total de 103 pacientes que cumplían con los criterios de inclusión, existiendo una razón de mujeres: hombres de 2,22, con edad promedio de 69 años.

$\mathrm{Al}$ analizar las características generales de la población estudiada (tabla 1) se observó un predominio del sexo femenino del 68,9\% ( $\mathrm{n}=71$ pacientes). En cuanto a la presencia de otros FRCV: el $75,7 \%(\mathrm{n}=78)$ de los pacientes presentó dislipemia, y el 88,3\% ( $\mathrm{n}=91)$ presentó sobrepeso y/u obesidad, según los criterios anteriormente establecidos. En la figura 1 , se observa también la prevalencia de otros FRCV, como tabaquismo y DM (tabla 2). 


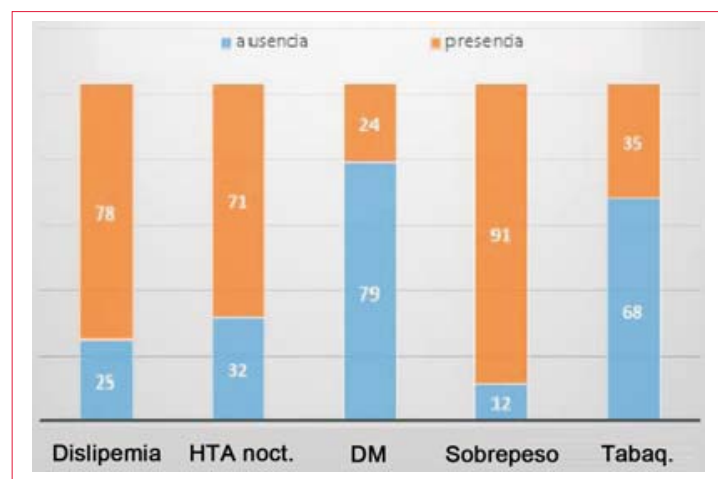

Figura 1. Factores de riesgo cardiovascular. HTA noct: hipertensión arterial nocturna; DM: diabetes mellitus; Tabaq: tabaquismo.

\begin{tabular}{|c|c|c|}
\hline & $\begin{array}{c}\text { Frecuencia } \\
\text { absoluta }\end{array}$ & $\begin{array}{l}\text { Frecuencia } \\
\text { relativa } \%\end{array}$ \\
\hline \multicolumn{3}{|l|}{ Dislipemia } \\
\hline Ausencia & 12 & 16,9 \\
\hline Hipercolesterolemia & 15 & 21,1 \\
\hline Hipertrigliceridemia & 14 & 19,7 \\
\hline Mixta & 30 & 42,3 \\
\hline \multicolumn{3}{|l|}{ MAPA } \\
\hline Dipper & 31 & 43,7 \\
\hline Non dipper & 32 & 45,1 \\
\hline Riser & 8 & 11,3 \\
\hline Extreme dipper & - & - \\
\hline Diabetes mellitus & 20 & 28,2 \\
\hline \multicolumn{3}{|l|}{ IMC } \\
\hline Normopeso & 9 & 12,7 \\
\hline Sobrepeso & 20 & 28,2 \\
\hline Obeso & 42 & 59,2 \\
\hline Tabaquismo & 24 & 33,8 \\
\hline
\end{tabular}

Se destaca que, del total de pacientes incluidos, $68,9 \%(n=71)$ presenta HTA nocturna, factor determinante en la estratificación del riesgo CV.

Con respecto a los pacientes dislipémicos predomina la dislipemia mixta en un $37,9 \%(n=39)$. Al valorar el patrón de variabilidad en el MAPA se observó la siguiente distribución: dipper 49,5\%, non dipper $38,8 \%$, riser $7,8 \%$, extreme dipper $3,9 \%$.

Al comparar pacientes con HTA nocturna versus pacientes sin HTA nocturna, se encontró que la proporción de dislipémicos era ampliamente mayor

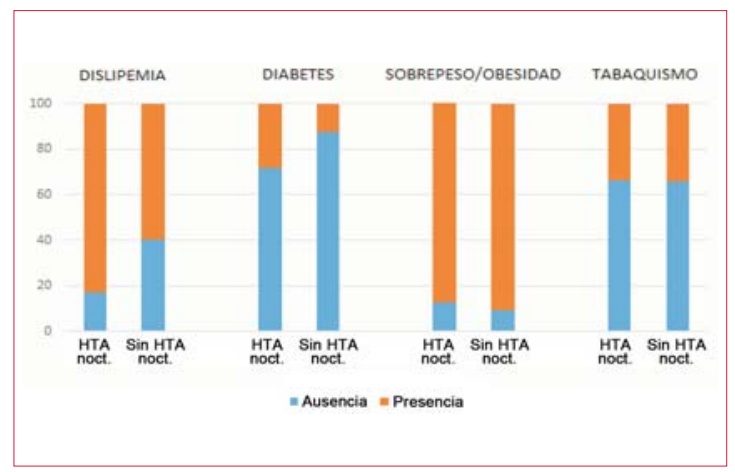

Figura 2. Comparación grupos con y sin HTA nocturna. HTA noct: hipertensión arterial nocturna.

en el primer grupo de pacientes, en un porcentaje de $83,1 \%, p=0,009$, estableciendo así una asociación estadísticamente significativa entre dislipemia e HTA nocturna. Se estimó OR=3,364 con un intervalo de confianza del 95\% (IC95\%): 1,315-8,609 (figura 2).

Si bien el porcentaje de pacientes con DM dentro del grupo HTA nocturna es mayor que en el grupo sin HTA nocturna $(28,2 \%$ vs. $12,5 \%)$ no pudo establecerse una asociación estadísticamente significativa entre ambas variables, $\mathrm{p}>0,05$ (figura 2 ).

Se compararon los tipos de perfil lipídico en los pacientes con y sin HTA nocturna, no encontrándose diferencia entre ambas poblaciones. La dislipemia mixta se presenta en mayor proporción en ambos grupos. La hipertrigliceridemia, al igual que la hipercolesterolemia, se presentan en similar proporción en ambos grupos (figura 3).

No se encontró una asociación estadísticamente significativa entre el tipo de dislipemia y el patrón de variabilidad para cada paciente $(\mathrm{p}=0,127)$ (tabla 3). Es decir, no se puede inferir el patrón de variabilidad conociendo el perfil de dislipemia. Puede observarse que para todas las categorías de dislipemia el patrón predominante fue el patrón dipper. En aquellos pacientes con hipertrigliceridemia se destaca el aumento de la proporción de patrón riser.

$\mathrm{Al}$ evaluar pacientes hipertensos con dislipemia no se pudieron inferir los patrones de variabilidad en el MAPA. Sin embargo, observamos un aumento del patrón non dipper en pacientes con dislipemia e HTA nocturna versus pacientes que no tienen dislipemia o HTA nocturna, en una relación significativa (tabla 4 , figura 4 ).

\section{Discusión}

La HTA es el principal FR para mortalidad y/o discapacidad a nivel mundial ${ }^{(1,10)}$. Sin embargo, a pesar de la indudable jerarquía de la HTA, la compleja interacción fisiopatológica y metabólica entre los dis- 


\begin{tabular}{|lccccc|}
\hline \multicolumn{5}{l}{ Tabla 3. Relación entre dislipemia y patrones de MAPA. } \\
\cline { 2 - 5 } & Dipper & Non dipper & Riser & Extreme dipper & Total \\
\hline Dislipemia & $48,0(12)$ & $40,0(10)$ & $4,0(1)$ & $8,0(2)$ & $100(25)$ \\
NO & $55,0(11)$ & $40,0(8)$ & $5,0(1)$ & - & $100(20)$ \\
Hipercolesterolemia & $42,1(8)$ & $31,6(6)$ & $26,3(5)$ & - & $100(19)$ \\
Hipertrigliceridemia & $51,3(20)$ & $41,0(16)$ & $2,6(1)$ & $5,1(2)$ & $100(39)$ \\
Mixta & & & & \\
\hline
\end{tabular}

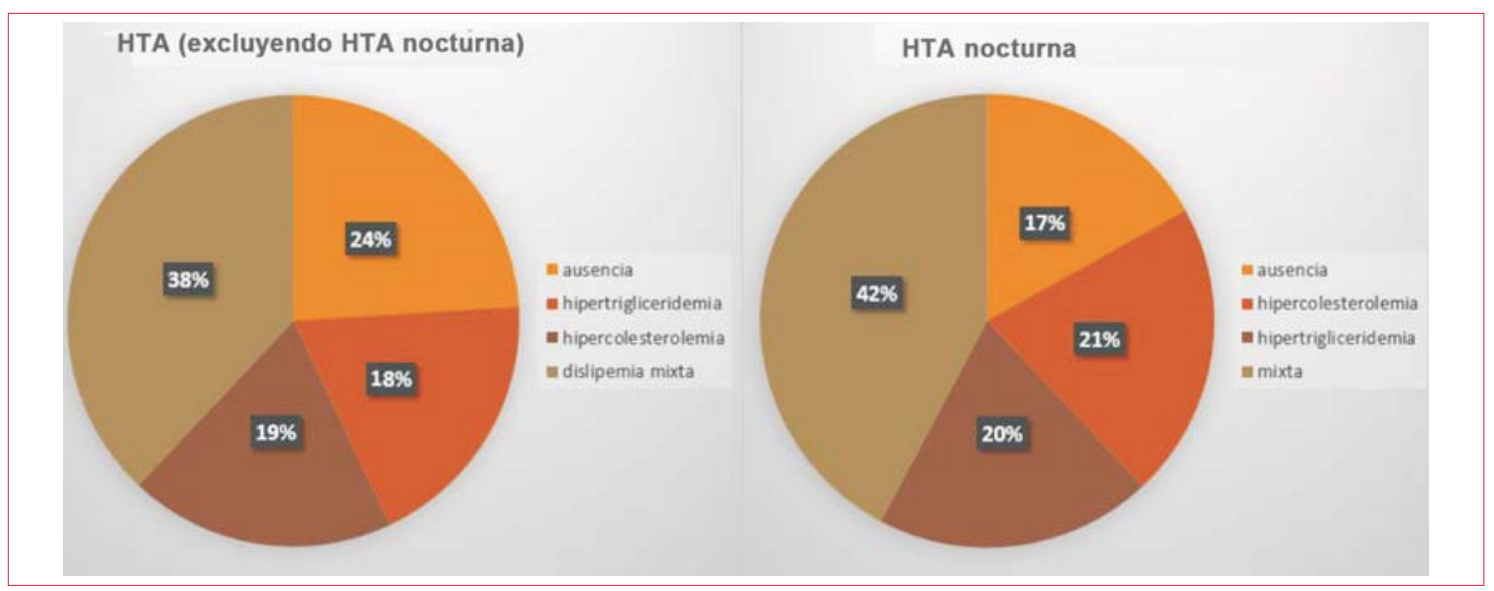

Figura 3. Comparación del perfil lipídico en pacientes con y sin HTA nocturna. HTA: hipertensión arterial.

tintos FRCV conduce a un abordaje interdisciplinario. Esta afirmación se refleja en nuestra serie, con $75,7 \%$ de pacientes dislipémicos, y un $88,3 \%$ con sobrepeso y/u obesidad.

El porcentaje de pacientes dislipémicos hallados fue superior al encontrado en series internacionales, pudiendo deberse a que los pacientes que concurren a nuestra policlínica son en un alto porcentaje $(67,2 \%)$ hipertensos severos de difícil control. Este porcentaje supera al obtenido por Toledo y colaboradores $(64,8 \%)$, entre 2015 y $2017^{(11)}$.

Se destaca también un alto porcentaje $(88,3 \%)$ de pacientes con sobrepeso y/u obesidad, el cual es levemente superior al obtenido por Toledo y colaboradores $(87,7 \%)^{(11)}$.

En cuanto al perfil lipídico, predomina la dislipemia mixta en 37,9\% ( $n=39)$. Con respecto al patrón de variabilidad en el MAPA, la población se distribuyó de la siguiente manera: dipper 49,5\%, non dipper $38,8 \%$, riser 7,8\%, extreme dipper 3,9\% (figura 5).

En nuestro estudio, como indicadores de riesgo $\mathrm{CV}$, utilizamos las siguientes variables: HTA nocturna y patrón de variabilidad non dipping.

Observamos que el 68,9\% de la población general presentaba HTA nocturna. Se encontró una asociación estadísticamente significativa entre

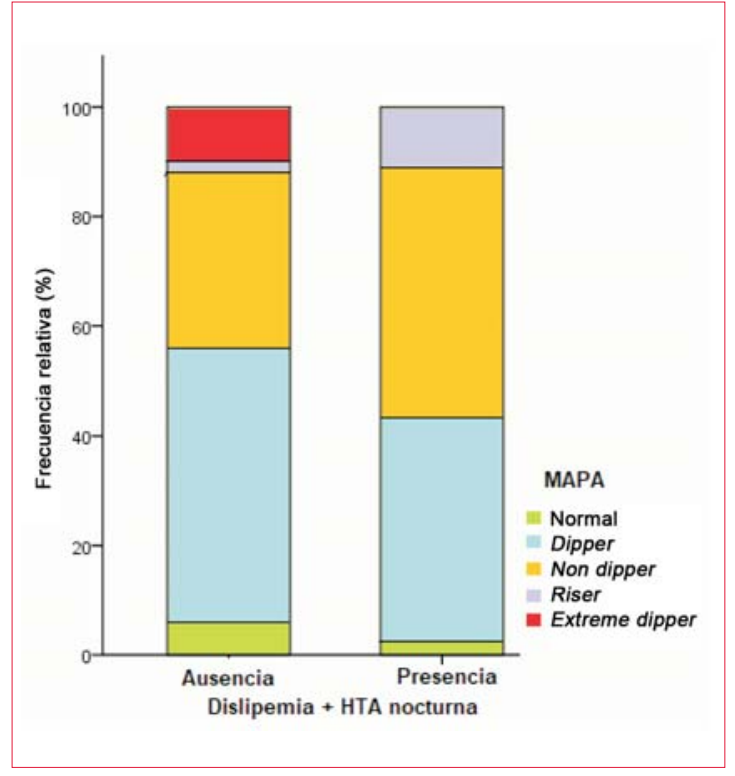

Figura 4. Diagrama de barras para MAPA según población con dislipemia e HTA nocturna. MAPA: monitoreo ambulatorio de la presión arterial.

dislipemia e HTA nocturna. En particular se observa que, en los pacientes con HTA nocturna, la proporción de dislipémicos es de $83,1 \%,(p=0,009)$; $\mathrm{OR}=3,364$. 


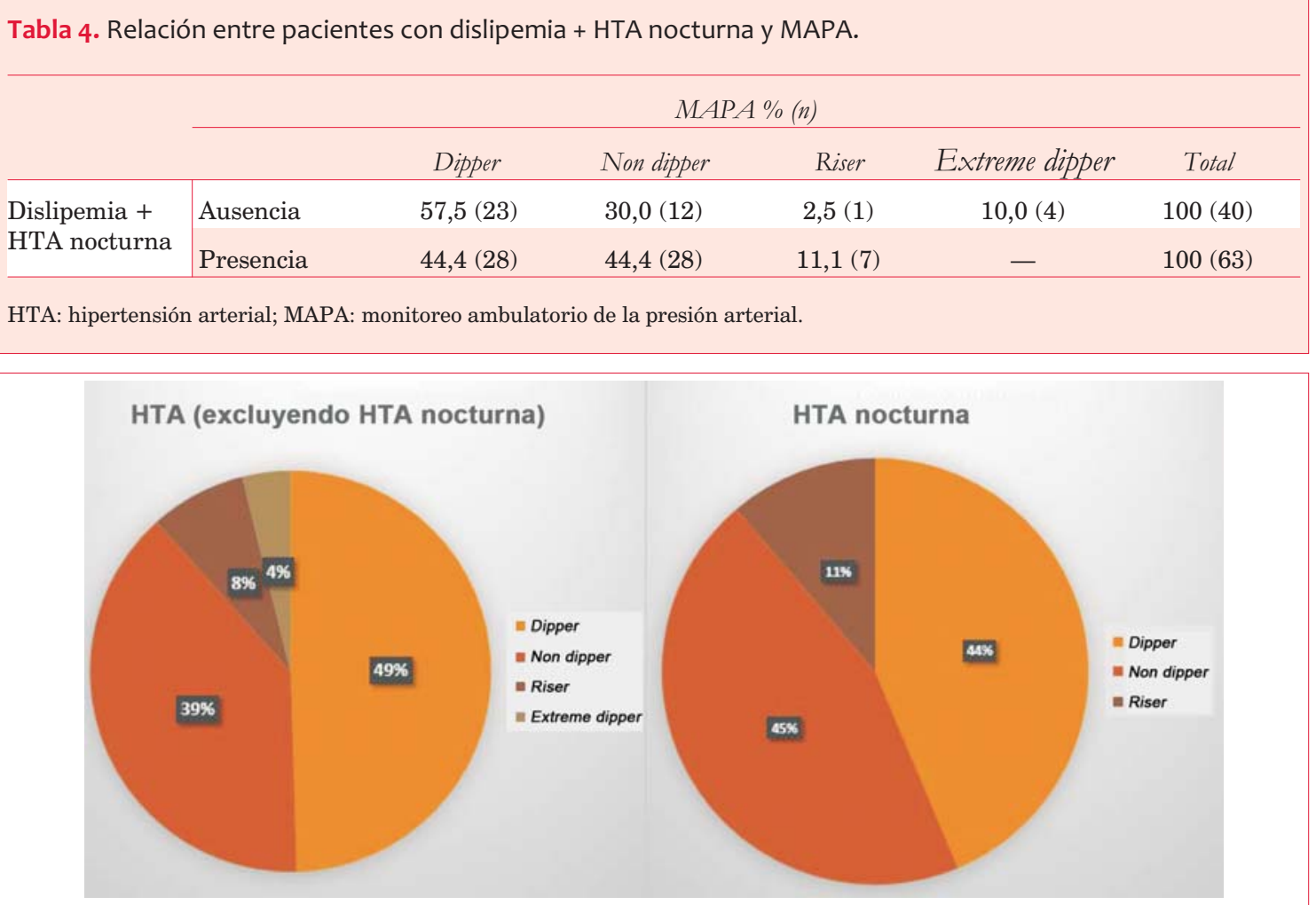

Figura 5. Patrón de variabilidad en pacientes con y sin HTA nocturna. HTA: hipertensión arterial.

La monitorización de PA ambulatoria ha demostrado ser mejor predictor que la PA basal(2).

Un estudio publicado por Yang y colaboradores en JAMA en 2019, demuestra que la HTA en 24 horas y la HTA nocturna aumentan el riesgo de muerte y de eventos CV, y que este es aún más alto si se asocian ambas variables ${ }^{(12)}$.

Otro dato importante que coincide con estudios internacionales, como el publicado en la Revista Americana de Hipertensión en 2014 por De La Sierra y colaboradores, es el incremento de la proporción de pacientes non dipper en el grupo de pacientes con HTA nocturna. Mientras que en la población general de hipertensos, el patrón de variabilidad predominante es el patrón dipper en un 49,5\%, en los pacientes con HTA nocturna asciende a primer lugar el patrón non dipper en un 45,1\% vs. 43,7\% para el patrón dipper (tablas 1 y 2 , gráfico 5 ). El estudio publicado por De La Sierra, demuestra que tanto la HTA nocturna como el patrón non-dipping son indicadores de un perfil CV de mayor riesgo, incluyendo FRCV tradicionales como edad, DM, obesidad, daño orgánico, hipertrofia ventricular izquierda, microalbuminuria, disminución de la función renal e historia previa de eventos $\mathrm{CV}^{(13)}$.

Si comparamos a los pacientes con HTA nocturnay dislipemia con los que no presentan ambas variables, observamos que existe una asociación significativa en- tre dicho grupo y los patrones de variabilidad que se relacionan con mayor riesgo $\mathrm{CV}$ (non dipping y riser). Es decir, la HTA nocturna, el patrón non dipping y la dislipemia se encuentran ampliamente relacionados, determinando un incremento en el riesgo CV.

Por lo tanto, aquellos pacientes que asocian HTA y dislipemia se caracterizan por presentar indicadores de mayor riesgo CV. La simple asociación de FRCV (síndrome metabólico) incrementa el riesgo $\mathrm{CV}$, siendo la asociación entre HTA y dislipemia mucho más frecuente de lo que pensábamos en nuestro medio. La explicación fisiopatológica se sustenta en disfunción endotelial, activación del sistema simpático y del sistema renina-angiotensina-aldosterona y al aumento de expresión de receptores de angiotensina y endotelina a nivel renal. El aumento de LDLc favorece la disfunción endotelial, lo que conlleva a una disminución de la síntesis de óxido nítrico (NO) con la consiguiente desaparición de sus efectos vasodilatadores, antiinflamatorios y antioxidantes. Las partículas de LDLc oxidadas incrementan la expresión de receptores de angiotensina I (AT1) lo que favorece la reabsorción de sodio y agua a nivel renal, la vasoconstricción y la disminución del NO. A su vez, se ha demostrado un aumento de los receptores de endotelina y AT1 a nivel renal y sus consecuentes efectos vasoconstrictores.

Es así que los FRCV comparten vías metabólicas comunes que incrementan el riesgo $\mathrm{CV}$, dificultando 
el control de los mismos por separado, debiéndose abordar el tratamiento del paciente HTA en base al riesgo CV y no a las cifras de PA únicamente, tal como recomiendan las guías internacionales de $\operatorname{HTA}^{(14,15)}$.

\section{Limitaciones}

Debemos señalar principalmente el escaso número de pacientes. En cuanto a las características de la población en estudio, debe destacarse el porcentaje elevado de pacientes con HTA grado III e IMC elevado, lo que puede sesgar los resultados.

\section{Conclusiones}

El control de los FRCV requiere de abordaje multidisciplinario dada su compleja fisiopatología e interacción. Un alto porcentaje de pacientes hipertensos analizados en este estudio presentan concomitantemente dislipemia. La HTA nocturna se asocia de forma significativa con dislipemia y patrón non dipper.

Contribución de autores

María Noel Rivero,

https://orcid.org/0000-0003-1306-9480. Recolección de datos, redacción, tablas, correcciones y respuesta a editores. Luca Quiroz, https://orcid.org/0000-0002-0958-2508. Recolección de datos, redacción, tablas y correcciones. Paola Spósito, https://orcid.org/0000-0001-8715-8385. Supervisión del trabajo, redacción y corrección final. Álvaro Huarte, https://orcid.org/0000-0002-9053-8140. Corrección final.

Este artículo fue aceptado para su publicación por: Editor jefe Dr. Gerardo Soca.

\section{Bibliografía}

1. Mills KT, Stefanescu A, He J. The global epidemiology of hypertension. Nat Rev Nephrol. 2020; 16 (4):223-37. doi:10.1038/s41581-019-0244-2

2. Williams B, Mancia G, Spiering W, Agabiti, Rosei E, Azizi M, et al. $2018 \mathrm{ESC} / \mathrm{ESH}$ guidelines for the management of arterial hypertension. Eur Heart J. 2018; 39(33):3021-104. doi: 10.1093/eurheartj/ ehy339

3. Zeron RMC, Albuquerque VC. Hypertension and cholesterol. Rev Assoc Med Bras (1992). 2019;65(12): 1421-2. doi:10.1590/1806-9282.65.12.1421

4. Piepoli MF, Hoes AW, Agewall S, Albus C, Brotons C, Cataplano AL, et al. 2016 European Guidelines on cardiovascular disease prevention in clinical practice: the Sixth Joint Task Force of the European Society of Cardiology and Other Societies on Cardiovascular Disease Prevention in Clinical Practice (constituted by representatives of 10 societies and by invited experts)Developed with the special contribution of the
European Association for Cardiovascular Prevention \& Rehabilitation (EACPR). Eur Heart J. 2016;37 (29):2315-81. doi:10.1093/eurheartj/ehw106

5. Rodriguez Roca GC, Alonso-Moreno FJ, Barrios V, Llisterri JL, Lou S, Matalí A, et al. Característica de la presión arterial en una población de dislipémicos española asistida en atención primaria. Estudio LIPICAPPA. Rev. Esp Cardiol. 2007; 60(8): 825-32. doi: 10.1157/13108996

6. Manual nacional de abordaje del tabaquismo en el primer nivel de atención [Internet]. Montevideo: MSP; 2009 [consulta: 30 Oct 2020]. Disponible en: https://www.who.int/fctc/reporting/Annexsixurue.pdf

7. Davies MJ, D'Alessio DA, Fradkin J, Kernan WN, Mathieu C, Mingrone G, et al. Management of hyperglycemia in type 2 diabetes, 2018. A consensus report by the American Diabetes Association (ADA) and the European Association for the Study of Diabetes (EASD). Diabetes Care 2018;41(12): 2669701. doi: $10.2337 /$ dci18-0033

8. Moreno M. Definición y clasificación de obesidad. Rev Med Clin Condes 2012; 23(2):124-8.

9. Mach F, Baigent C, Catapano A, Koskinas K, Casula M, Badimon L et al. 2019 ESC/EAS Guidelines for the management of dyslipidaemias: lipid modification to reduce cardiovascular risk. Eur Heart J. 2020;41(1):111-88. doi: 10.1093/eurheartj/ehz455.

10. Lim SL, Vos T, Flaxman AD, Danaei G, Shibuya K, Adair- Rohani H, et al. A comparative risk assessment of burden of disease and injury attributable to 67 risk factors and risk factor clusters in 21 regions, 1990-2010: a systematic analysis for the Global Burden of Disease Study 2010. Lancet 2012; 380(9859): 2224-60. doi:10.1016/S0140-6736(12)61 766-8.

11. Toledo MV, Spósito P, Llorrens M. Casuística en pacientes asistidos en una policlínica. Rev Urug Cardiol. 2019;34(2):163-9. doi: 10.29277/cardio.34.2.7

12. Yang W-Y, Melgarejo JD, Thijs L, Zhang Z-Y, Boggia J, Wei F-F. Association of office and ambulatory blood pressure with mortality and cardiovascualr outcome. JAMA.2019; 322 (5):409-20. doi: 10.1001/jama.2019.9811

13. de la Sierra A, Gorostidi M, Baneja JR, Segura J, de La Cruz JJ, Rui Lope LM. Nocturnal Hypertension or non dipper: which is better associated with the Cardiovascular Risk profile? Am J Hyperten.2014; 27(5): 680-7. doi:10.1093/ajh/hpt175

14. Mendizábal Y, Llorens S, Nava E. Hypertension in metabolic syndrome: vascular pathophysiology. Int J Hypertens. 2013;2013:1-15. doi:10.1155/2013/2308

15. Marotta M, Vía D. Disfunción endotelial. En: Artagaveytia N, Bianchi S, Cayota A. Tema de patología médica. Montevideo: Oficina del Libro; 2017. P.463-508. 